\title{
Satisfação e acesso à saúde bucal das pessoas que vivem com HIV/Aids no nordeste brasileiro
}

\author{
Access to and satisfaction with oral health care among persons with \\ HIV/Aids in Northeastern Brazil
}

Lizaldo Andrade Maia', Anya Pimentel Gomes Fernandes Vieira-Meyer $\mathbf{2 , 3}$, Maria Vieira de Lima

Saintrain $\mathbf{4}$, Sharmênia de Araújo Soares Nuto ${ }^{\mathbf{3}, \mathbf{4}}$, Ana Patrícia Pereira Morais $\mathbf{5}$

DOI: $10.1590 / 0103-1104202112912$

RESUMO Este estudo objetivou avaliar, na perspectiva das Pessoas Vivendo com HIV/Aids (PVHA), uso e satisfação com os serviços públicos de saúde bucal no Sistema Único de Saúde em Fortaleza (CE). Aplicaram-se questionários estruturados sobre perfil socioeconômico, uso e satisfação dos serviços públicos de saúde bucal para 241 PVHA que frequentam 8 Serviços de Assistência Especializada em HIV/ Aids. A idade média dos usuários foi de 37,8 \pm 9,6 anos; 161 (68,3\%) do sexo masculino; com ensino médio completo ( $\mathrm{n}=79 ; 32,8 \%) ; 59$ (24,5\%) ganham até 1 salário mínimo (US\$255). Apenas 155 (64,3\%) foram ao dentista nos dois anos anteriores. Destes, 68 (28,2\%) frequentavam serviços públicos, 31 (45,6\%) dos quais não completaram o tratamento por falta de materiais/equipamentos defeituosos/reformas nas unidades de saúde. A nota média atribuída pelo paciente ao atendimento dos profissionais foi 7,6 $( \pm 2,5), 50(73,6 \%)$ declararam-se muito satisfeitos/satisfeitos. Quanto ao atendimento humanizado, 59 (86,7\%) estavam muito satisfeitos/satisfeitos. Pacientes encaminhados pelo Serviços de Assistência Especializada em HIV/ Aids e os que residem perto das unidades de saúde tiveram probabilidade significativamente maior de usar os serviços públicos. Apesar do uso limitado dos serviços públicos de saúde bucal, principalmente devido ao acesso insuficiente e aos procedimentos ineficazes de agendamento, os serviços usados pelos entrevistados foram avaliados satisfatoriamente.

PALAVRAS-CHAVE Saúde bucal. Acesso aos serviços de saúde. HIV.

1 Secretaria Municipal de Saúde de Fortaleza (SMSF) - Fortaleza (CE), Brasil.

lizaldo@gmail.com

${ }^{2}$ Centro Universitário Christus (Unichristus) Fortaleza (CE), Brasil.

${ }^{3}$ Fundação Oswaldo Cruz (Fiocruz) - Fortaleza (CE), Brasil.

4 Universidade de Fortaleza (Unifor) - Fortaleza (CE), Brasil.

5 Universidade Estadual do Ceará (Uece) - Fortaleza (CE), Brasil.

\begin{abstract}
This study aimed to investigate, from the perspective of People Living with HIV/Aids (PLWHA), the use of and satisfaction with public oral health services within the Unified Health System (SUS) in Fortaleza (Northeastern Brazil). Structured questionnaires on socioeconomic profile and public oral health service use and satisfaction were administered to 241 PLWHA attending eight Specialized Healthcare Services (SAE) in HIV/Aids. The mean age was $37.8 \pm 9.6$ years, 161 (68.3\%) were male, 79 (32.8\%) had completed high school, and 59 (24.5\%) reported earning $\leq 1$ minimum wage (USD 225). Only 155 ( $64.3 \%$ ) had been to the dentist in the preceding 2 years. Of these, 68 (28.2\%) attended public services, but nearly half (45.6\%) did not complete treatment due to lack of supplies, malfunctioning equipment or ongoing repair of facilities. On average, the service was graded 7.6 \pm 2.5 , and 50 PLWHA (73.6\%) reported being satisfied/very satisfied. As for humanized care, $86.7 \%$ were satisfied/very satisfied. Patients referred by SAE or residing near the facility were significantly more likely to use public services. Despite the limited use of public oral health services, mainly due to insufficient access and ineffective appointment scheduling and referral procedures, the services were mostly graded as satisfactory.
\end{abstract}

KEYWORDS Oral health. Access to health services. HIV. 


\section{Introdução}

Com a introdução da terapia antirretroviral para a Síndrome da Imunodeficiência Adquirida (Aids) no final dos anos 1990, a expectativa e a qualidade de vida das Pessoas Vivendo com HIV/Aids (PVHA) melhoraram consideravelmente. Além disso, o número de novas infecções por Vírus da Imunodeficiência Humana (HIV) em todo o mundo diminuiu 19\% na última década'. Apesar desses avanços, o HIV/Aids continua sendo um sério problema de saúde pública, principalmente devido à sua alta prevalência e a altos índices de morbimortalidade ${ }^{2,3}$.

Em todo o mundo, havia 37,9 milhões de PVHA em 20184. No Brasil, de 1980 a 2019, foram identificados 966.058 casos de Aids; e 338.905 pessoas morreram tendo o HIV/Aids como causa básica entre 1980 e $2018^{3}$. No entanto, o atendimento integrado de PVHA - desde medidas de promoção da saúde e prevenção de agravos até recuperação e acompanhamento multidisciplinar - ainda é um desafio no cenário da atenção à saúde pública ${ }^{5}$, apesar da forte legislação apoiando essa população ${ }^{6}$.

É importante enfatizar que a promoção da saúde é conceituada como determinante e condicionante da saúde por se preocupar com o modo de viver da população, fatores econômicos, ambientais, ecológicos e culturais; incorporando iniciativas políticas orientadas 7,8. A prevenção de agravos, por sua vez, busca a ausência de doença, com enfoque no conhecimento epidemiológico, objetivando o controle da transmissão e a redução do risco de agravos à saúde 9 .

A crescente sobrevivência das PVHA implica uma demanda crescente por atenção à saúde bucal. $\mathrm{O}$ treinamento e a disposição dos dentistas para tratar as PVHA são essenciais para $\mathrm{o}$ atendimento integrado, principalmente por causa da manifestação precoce de lesões relacionadas com a Aids na cavidade oral. Tais lesões são vistas em até $80 \%$ dos pacientes com essa síndrome, sendo um importante marcador de imunossupressão ${ }^{10}$.
Criado em 1988, ancorado nos princípios da universalidade, equidade e atendimento integrado, o Sistema Único de Saúde (SUS) oferece atenção primária, secundária e terciária a todas as pessoas ${ }^{11}$. O SUS é o primeiro sistema de saúde de um país em desenvolvimento a proporcionar acesso gratuito e universal para tratamento das PVHA, incluindo a distribuição gratuita de medicamentos antirretrovirais de forma sistemática ${ }^{6}$.

Na década de 1990, o Ministério da Saúde incentivou os governos estaduais e municipais a implantarem o Serviço de Assistência Especializada em HIV/Aids (SAE), que é responsável pelo acompanhamento dos pacientes, inclusive com encaminhamentos de pacientes HIV positivos para atendimento odontológico na atenção primaria e dispensação da medicação antirretroviral ${ }^{12}$.

O SUS fornece uma ampla gama de serviços de Atenção Primária à Saúde (APS) sob a égide da Estratégia Saúde da Família (ESF). O acesso aos serviços de APS é realizado por Equipes de Saúde da Família (EqSF), que incluem médico, enfermeiro, auxiliar de enfermagem e agentes comunitários de saúde. Desde o ano 2000, a ESF também é atendida por Equipes de Saúde Bucal (ESB) ${ }^{13}$. Cada ESF/ESB, instalada nos Centros de Saúde da Família (CSF), cobre uma área geográfica específica ${ }^{14} \mathrm{e}$, se necessário, encaminha os pacientes para os Centros de Especialidades Odontológicas (CEO). Em 2019, as ESF cobriam 96\% dos municípios brasileiros, correspondendo a $64,2 \%$ da população. Da mesma forma, no mesmo ano, 28.797 ESB estavam implementadas em todo o País, correspondendo a uma cobertura de $42,8 \%$ da população ${ }^{15}$. No município de Fortaleza, Ceará (CE), além dos 8 SAE, a Rede de Atenção, em 2019, era composta de 109 CSF, 10 hospitais públicos municipais (atenção secundária e terciária), 9 Unidades de Pronto Atendimento (UPA); 11 Centros de Atenção Psicossocial (Caps), 4 CEO e 4 Policlínicas. Informações do Cadastro Nacional de Estabelecimento de Saúde (CNES) revelam uma cobertura da ESF no município de $56 \%$ (379 EqSF) e 33\% de Saúde Bucal (260 ESB) ${ }^{\mathbf{1 5}}$. 
Estudos têm mostrado baixos níveis médios de escolaridade e renda entre as PVHA $^{16,17}$, indicando que uma parte importante dessa população depende de serviços públicos para suas necessidades de saúde. No entanto, pouco se sabe sobre acesso, uso de serviços e satisfação com os serviços públicos de saúde bucal em países com sistemas universais de saúde gratuitos como o Brasil. Dessa forma, o objetivo deste estudo foi avaliar, na perspectiva das PVHA, o uso e a satisfação dos serviços públicos de saúde bucal em uma grande cidade brasileira.

\section{Material e métodos}

Estudo quantitativo, descritivo analítico, realizado em todos os SAE em Fortaleza (CE). Localizada na região Nordeste, é a quinta maior capital do Brasil (2,5 milhões de habitantes). Nessa região pobre, a taxa de detecção de Aids está acima da média nacional em 2018 (Fortaleza e Brasil, taxas de detecção 26,4 e 17,8 por 100 mil hab. respectivamente) $)^{3,18}$.

A população do estudo consistiu em 2.153 PVHA residentes em Fortaleza (CE) e registradas em todos os oitos SAE municipais, de acordo com dados secundários obtidos no Serviço de Vigilância Epidemiológica do município e na Coordenação de Doenças Sexualmente Transmissíveis/Aids/Hepatites virais. Com o nível de significância estatística estabelecido em $90 \%$ e o erro amostral em $5 \%$, foi calculado um tamanho amostral de 241 indivíduos.

Para inclusão como participante do estudo, era necessário ser PVHA, usuário de um dos SAE em Fortaleza (CE). As PVHA foram recrutadas proporcionalmente em todos os oito SAE. Após consulta de rotina aos infectologistas, o paciente era questionado se poderia participar da pesquisa pelo médico. Esses profissionais previamente contatados pelo pesquisador, sendo sensibilizados a apoiar a pesquisa. Todos os infectologistas aceitaram participar na captação de pacientes para o presente estudo. Os pacientes eram encaminhados ao pesquisador após aceite em participar da pesquisa. Os pacientes foram excluídos da amostra se: i) residiam fora de Fortaleza (CE); ii) tinham menos de 18 anos; iii) estavam em sua primeira consulta; e iv) tinham diagnóstico de HIV há menos de dois anos. As informações foram coletadas por meio de questionário estruturado, aplicado presencialmente em 2013 por um único pesquisador em uma sala reservada, englobando questões sobre o nível socioeconômico, uso e satisfação do serviço público de saúde bucal, cuidado humanizado e os processos de assistência à saúde, conforme definido por Donabedian ${ }^{19}$.

No software estatístico Epidata 3.1., construiu-se o banco de dados, sendo processado e analisado no Statistical Package for Social Science (SPSS) 19.0 for Windows (SPSS Inc, Chicago, IL, USA). Os resultados descritivos foram processados por meio de frequências simples e percentuais. Além disso, a análise das associações entre as variáveis categóricas realizou-se mediante referência cruzada, calculando o teste Qui-quadrado de Pearson $\left(\mathrm{x}^{2}\right)$. O valor de $\mathrm{p}$ foi considerado significativo quando menor ou igual a 0,05.

Todos os participantes foram informados dos objetivos e da metodologia do estudo e assinaram o Termo de Consentimento Livre e Esclarecido. A pesquisa respeitou todos os preceitos éticos da Resolução $n^{\circ} 466 / 2012$, tendo sido aprovado pelo Comitê de Ética em Pesquisa da Universidade Estadual do Ceará sob o número 357.459.

\section{Resultados}

Um total de 241 PVHA, distribuídas proporcionalmente nos 8 SAE de Fortaleza (CE), foi incluído na pesquisa. Sete sujeitos recusaram participação. A idade média foi de 37,8 $\pm 9,6$ anos (variação: 18-65). A maioria era do sexo masculino ( $\mathrm{n}=161 ; 68,3 \%)$, parda $(\mathrm{n}=183$; $75,9 \%)$, solteira ( $n=152 ; 63,1 \%)$, com ensino médio completo ( $\mathrm{n}=79 ; 32,8 \%)$, sem filhos 
( $\mathrm{n}=126 ; 52,3 \%)$. Sobre as condições econômicas, 49 (20,3\%) não têm renda, 59 (24,5\%) ganham até um salário mínimo e 83 (34,4\%) recebem entre um e dois salários mínimos - no momento da redação deste artigo, o salário mínimo oficial era equivalente a $\mathrm{R} \$ 998,00$ (US\$255) por mês.

Quanto ao uso do serviço público de saúde bucal, $122(50,6 \%)$ relataram receber tratamento odontológico em clínicas particulares, 78 (32,4\%) possuíam plano de saúde, 68 (28,2\%) frequentavam serviços públicos (CSF/ CEO), 44 (18,3\%) usavam serviços prestados por sindicatos, $16(6,6 \%)$ foram atendidos na Faculdade de Odontologia e 5 (2,0\%) possuíam seguro funeral com cobertura odontológica. Apenas 155 (64,3\%) dos entrevistados foram ao dentista nos últimos 2 anos, $83(34,4 \%)$ foram ao dentista há mais de 2 anos e $3(1,2 \%)$ nunca receberam atenção em saúde bucal. No primeiro desses subgrupos, 120 (77,4\%) eram consultas de rotina; 19 (12,6\%), emergências; e $16(10,3 \%)$, atendimento especializado.

Os entrevistados relataram frequentar serviços públicos como CSF ( $\mathrm{n}=61 ; 25,3 \%$ ) e CEO ( $n=7 ; 2,9 \%)$ ou outros serviços ( $=173$; $71,8 \%$ ). Metade ( $\mathrm{n}=34 ; 50 \%$ ) das PVHS que utilizam serviços públicos (CSF/CEO) o fez por demanda espontânea, enquanto 19 $(27,9 \%)$ foram encaminhadas pelo SAE. Quase dois terços $(64,7 \% ; n=44)$ dos pacientes que utilizam o sistema público de saúde informaram que tiveram seu problema de saúde bucal resolvido. A tabela 1 mostra a avaliação dos entrevistados que utilizaram o sistema público de saúde em relação à facilidade de acesso, resolução de problemas e finalização do tratamento $(\mathrm{n}=68)$.

Tabela 1. Forma de acesso, resolução do problema e tratamento completo nos serviços de saúde bucal (CSF/CEO). Informações das PVHA cadastradas nos SAE municipais. Fortaleza (Ceará, Brasil) - n=68

\begin{tabular}{|c|c|c|}
\hline & $\mathbf{n}$ & $\%$ \\
\hline \multicolumn{3}{|l|}{ Como conseguiu acesso à consulta? } \\
\hline Referenciado pelo SAE & 19 & 27,9 \\
\hline Por meio do agendamento com o ACS ou ASB & 3 & 4,4 \\
\hline Fichas distribuídas no dia & 34 & 50,0 \\
\hline Outros & 12 & 17.7 \\
\hline \multicolumn{3}{|l|}{ Está satisfeito com esta forma que conseguiu acesso à consulta? } \\
\hline $\operatorname{Sim}$ & 56 & 82,4 \\
\hline Não & 10 & 14,7 \\
\hline Em parte & 2 & 2,9 \\
\hline \multicolumn{3}{|l|}{ Considera que seu problema foi resolvido? } \\
\hline Sim & 44 & 64,7 \\
\hline Não & 21 & 30,9 \\
\hline Em parte & 3 & 4,4 \\
\hline \multicolumn{3}{|l|}{ O dentista completou o tratamento? } \\
\hline $\operatorname{Sim}$ & 37 & 54,4 \\
\hline Não & 31 & 45,6 \\
\hline \multicolumn{3}{|l|}{ Por que o dentista não completou o tratamento? } \\
\hline Falta material, problema no equipamento ou CSF em reforma & 18 & 58,1 \\
\hline Dentista faltou ao trabalho & 2 & 06,4 \\
\hline
\end{tabular}


Tabela 1. (cont.)

\begin{tabular}{lrr}
\hline & $\mathbf{n}$ & $\%$ \\
\hline Dentista não agendou o retorno & 4 & 12,9 \\
Paciente faltou à consulta e não foi remarcar & 3 & 9,7 \\
Dentista soube que usuário era HIV+, disse que procurasse outro CSF & 4 & 12,9 \\
\hline Fonte: Elaboração própria. & \\
CSF = Centro de Saúde da Família; CEO = Centro de Especialidades Odontológicas; SAE = Serviços de Assistência Especializada em HIV/ \\
Aids; ACS = Agente Comunitário de Saúde; ASB = Auxiliar em Saúde Bucal.
\end{tabular}

Na tabela 2, estão os resultados da avaliação dos pacientes em uma subamostra de 39 pessoas que receberam atenção em saúde bucal em clínicas públicas (CSF/CEO) e privadas. Dois deles $(5,2 \%)$ consideraram CSF/CEO melhores ou muito melhores que as clínicas privadas, 19 (48,7\%) conceituaram CSF/CEO piores ou muito piores, enquanto 18 (46,1\%) julgaram as duas opções semelhantes. Muitos usuários dos serviços públicos de saúde relataram ter omitido sua condição de HIV positivo durante as consultas odontológicas ( $\mathrm{n}=16$; $23,5 \%)$. Quatorze (87,6\%) o fizeram por medo de discriminação ou negação da consulta.

Tabela 2. Participação em atividade educativa, comparação do serviço público com o particular e discriminação ou resistência por parte da equipe nos serviços de saúde bucal (CSF/CEO). Informações das PVHA cadastradas nos SAE municipais. Fortaleza (Ceará, Brasil) $-\mathrm{n}=68$

n

Participou de atividade educativa com equipe de saúde bucal?

\begin{tabular}{|c|c|c|}
\hline Sim & 18 & 26,5 \\
\hline Não & 50 & 73,5 \\
\hline Se sim, foi satisfatória? & 18 & 100,0 \\
\hline \multicolumn{3}{|c|}{$\begin{array}{l}\text { Se foi atendido em serviço particular (hospital, consultório, plano de saúde), como classificaria o atendimento } \\
\text { prestado no CSF/CEO?^ }\end{array}$} \\
\hline Muito melhor & 1 & 2,6 \\
\hline Melhor & 1 & 2,6 \\
\hline lgual & 18 & 46,1 \\
\hline Pior & 15 & 38,4 \\
\hline Muito pior & 4 & 10,3 \\
\hline \multicolumn{3}{|c|}{ Passou por alguma forma de discriminação/preconceito durante o atendimento? } \\
\hline Sim & 2 & 2,9 \\
\hline Não & 66 & 97,1 \\
\hline \multicolumn{3}{|c|}{$\begin{array}{l}\text { Você observou algum tipo de resistência por parte do dentista ou das auxiliares em atendê-lo ou durante o } \\
\text { atendimento? }\end{array}$} \\
\hline Sim & 4 & 5,9 \\
\hline Não & 64 & 94,1 \\
\hline
\end{tabular}


Tabela 2. (cont.)

\begin{tabular}{|c|c|c|}
\hline & $\mathbf{n}$ & $\%$ \\
\hline \multicolumn{3}{|c|}{ Omitiu alguma vez que é HIV+ durante o atendimento odontológico no CSF/CEO? } \\
\hline Sim & 16 & 23,5 \\
\hline Não & 52 & 76,5 \\
\hline \multicolumn{3}{|l|}{ Se sim, por que omitiu? } \\
\hline Receio de discriminação & 09 & 56,3 \\
\hline Receio de não ser atendido & 5 & 31,3 \\
\hline Dentista conhecia família do paciente & 1 & 6,3 \\
\hline Pensou que não deveria falar & 1 & 6,3 \\
\hline
\end{tabular}

A tabela 3 apresenta as respostas dos entrevistados em relação à localização, à estrutura física e à disponibilidade de suprimentos nas unidades públicas de saúde (CSF/CEO). Apenas 68 (28,2\%) compareceram a serviços públicos, 31 (45,6\%) não completaram o tratamento por várias razões, incluindo falta de materiais/suprimentos, equipamentos com defeito ou reformas na infraestrutura da unidade de saúde. Quase metade $(n=32$; $47,1 \%)$ considerou a estrutura física adequada, enquanto 28 (41,2\%) confirmaram a disponibilidade dos suprimentos necessários para o tratamento de sua condição.

Tabela 3. Estrutura física do consultório odontológico e distância deste a residência do usuário. Informações das PVHA cadastradas nos SAE municipais. Fortaleza (Ceará, Brasil) - n=68

\begin{tabular}{lrr}
\hline & $\mathbf{n}$ & $\%$ \\
\hline A estrutura física do consultório do CSF/CEO é adequada para o atendimento? & 32 & 47,1 \\
Sim & 15 & 22,1 \\
Não & 3 & 4,4 \\
Em parte & 18 & 26,5 \\
Não sei & 28 & 41,2 \\
\hline O consultório do CSF/CEO tem todos os materiais necessários para o atendimento? & 32,4 \\
Sim & 22 & 7,4 \\
Não & 5 & 19,1 \\
Em parte & 13 & 1,4 \\
Não sei & 38 & 55,9 \\
Nos últimos 12 meses, o serviço parou por falta de material ou de manutenção? & 1 \\
Sim & 29 & 42,6 \\
Não & 30 \\
Não sei & & 1,5 \\
\hline
\end{tabular}


Tabela 3. (cont.)

\begin{tabular}{|c|c|c|}
\hline & $\mathbf{n}$ & $\%$ \\
\hline \multicolumn{3}{|l|}{ Se sim, por quanto tempo? } \\
\hline Entre duas e quatro semanas & 2 & 5,3 \\
\hline Entre um e dois meses & 9 & 23,7 \\
\hline Entre dois e quatro meses & 13 & 34,2 \\
\hline Entre quatro e seis meses & 9 & 23,7 \\
\hline Entre seis meses e um ano & 3 & 7,9 \\
\hline Mais de um ano & 1 & 2,6 \\
\hline Não sei & 1 & 2,6 \\
\hline \multicolumn{3}{|c|}{ Mora no bairro perto do CSF/CEO em que foi atendido? } \\
\hline $\operatorname{Sim}$ & 42 & 61,8 \\
\hline Não & 26 & 38,2 \\
\hline \multicolumn{3}{|c|}{ Se não, por que do atendimento longe do bairro onde mora? } \\
\hline Solicitação do usuário & 11 & 42,3 \\
\hline Encaminhamento do SAE & 11 & 42,3 \\
\hline Outro & 4 & 15,4 \\
\hline \multicolumn{3}{|c|}{ Teve dificuldade de deslocamento de sua casa até o CSF/CEO em que foi atendido? } \\
\hline Sim & 9 & 13,2 \\
\hline Não & 59 & 86,8 \\
\hline
\end{tabular}

Fonte: Elaboração própria.

CSF = Centro de Saúde da Família; CEO = Centro de Especialidades Odontológicas; SAE = Serviços de Assistência Especializada em HIV/Aids.

Quando os usuários do CSF/CEO foram solicitados a classificar os cuidados recebidos (de 0 a 10), a pontuação média foi de $7,6 \pm 2,5$. Três quartos ( $\mathrm{n}=50 ; 73,6 \%)$ estavam satisfeitos/muito satisfeitos com o serviço, enquanto um quarto (n=18; 26,5\%) não estava muito satisfeito/insatisfeito. A tabela 4 mostra as respostas dos usuários do CSF/CEO em relação às práticas de cuidado humanizado observadas durante a consulta.
Para verificar a associação entre o uso do serviço público de atenção à saúde bucal e as variáveis das tabelas 1 a 4, os achados foram submetidos à tabulação cruzada e ao teste do qui-quadrado. Os resultados indicam que os pacientes encaminhados pelo SAE e os pacientes que residem perto das unidades de saúde tiveram uma probabilidade significativamente maior de usar os serviços públicos ( $\mathrm{p} \leq 0,001 \mathrm{e}$ $\mathrm{p} \leq 0,01$ respectivamente). 
Tabela 4. Dispositivos da humanização do cuidado nos serviços de saúde bucal (CSF/CEO). Informações das PVHA cadastradas nos SAE municipais. Fortaleza (Ceará, Brasil) - n=68

\begin{tabular}{|c|c|c|}
\hline & n & $\%$ \\
\hline \multicolumn{3}{|c|}{ O dentista está preparado para resolver os problemas de saúde bucal do paciente? } \\
\hline Sim & 57 & 83,8 \\
\hline Não & 6 & 8,8 \\
\hline Em parte & 1 & 1,5 \\
\hline Não sei & 4 & 5,9 \\
\hline \multicolumn{3}{|c|}{ Grau de satisfação geral do atendimento por parte do dentista } \\
\hline Muito satisfeito & 15 & 22,1 \\
\hline Satisfeito & 35 & 51,5 \\
\hline Pouco satisfeito & 10 & 14,7 \\
\hline Insatisfeito & 8 & 11,8 \\
\hline \multicolumn{3}{|c|}{ Em relação ao respeito e à atenção no atendimento por parte dentista } \\
\hline Muito satisfeito & 23 & 33,8 \\
\hline Satisfeito & 36 & 52,9 \\
\hline Pouco satisfeito & 6 & 8,8 \\
\hline Insatisfeito & 3 & 4,4 \\
\hline \multicolumn{3}{|c|}{ Em relação ao repasse de informações de forma clara durante o atendimento por parte do cirurgião-dentista } \\
\hline Muito satisfeito & 19 & 27,9 \\
\hline Satisfeito & 31 & 45,6 \\
\hline Pouco satisfeito & 11 & 16,2 \\
\hline Insatisfeito & 7 & 10,3 \\
\hline
\end{tabular}

Em relação ao acolhimento, a escutar a queixa, a compreender os medos, angústias, vulnerabilidade, por parte do dentista

\begin{tabular}{lrr} 
Muito satisfeito & 27 & 39,7 \\
Satisfeito & 28 & 41,2 \\
Pouco satisfeito & 5 & 7,4 \\
Insatisfeito & 8 & 11,8 \\
\hline
\end{tabular}

Em relação ao esforço do dentista em buscar interações com outros profissionais da EqSF ou ajuda de outros setores no sentido de atender a todas as suas necessidades de paciente

\begin{tabular}{lrr} 
Muito satisfeito & 4 & 5,9 \\
Satisfeito & 2 & 2,9 \\
Pouco satisfeito & 1 & 1,5 \\
Insatisfeito & 3 & 4,4 \\
Não sabe & 58 & 85,3 \\
\hline
\end{tabular}

Fonte: Elaboração própria.

CSF = Centro de Saúde da Família; CEO = Centro de Especialidades Odontológicas; SAE = Serviços de Assistência Especializada em HIV/Aids; EqSF = Equipe de Saúde da Família. 


\section{Discussão}

Devido à sua amplitude, este estudo permitiu uma melhor compreensão do uso e satisfação referente ao serviço público de saúde bucal entre as PVHA e alguns dos aspectos que podem moderar seu uso na perspectiva dessas pessoas. Além disso, o nível de satisfação das PVHA com a qualidade, acesso e resolutividade dos serviços possibilita o entendimento dos desafios enfrentados por sistemas universais gratuitos que englobam a atenção à saúde bucal, servindo de base para formulação de políticas públicas na área.

Considera-se a amostra desta investigação representativa da população geral das PVHA vivendo em Fortaleza (CE), pois foram entrevistadas onde os medicamentos antirretrovirais são dispensados. Devido ao alto custo dos medicamentos antirretrovirais e sua dispensação gratuita por meio do SUS, é improvável que uma proporção significativa de PVHA não usaria o sistema público de saúde para adquirir seus medicamentos.

$\mathrm{O}$ atendimento integrado por equipes multiprofissionais é necessário para minimizar o risco de complicações de saúde evitáveis ${ }^{2}$. No entanto, o acesso limitado das PVHA à atenção à saúde bucal é um problema sério, considerando que essas têm uma maior necessidade dessa atenção do que a população geral20,21. As necessidades não atendidas de atenção à saúde bucal são comuns entre as PVHA, e as barreiras ao atendimento parecem estar associadas à raça e a situação socioeconômi$\mathrm{ca}^{22}$. De fato, conforme demonstrado pelos indicadores socioeconômicos pesquisados, as necessidades de atenção à saúde bucal são substanciais nesses pacientes. Apenas um terço dos entrevistados cursou até o ensino médio, um quarto não possuía renda, e a maioria ganhava menos de dois salários mínimos. Esses achados são corroborados por outros estudos que apontam baixos níveis de escolaridade e renda entre as PVHA ${ }^{16,17}$. Devido a condições socioeconômicas e educacionais desfavoráveis, uma proporção significativa de PVHA depende exclusivamente do SUS e tem uma necessidade urgente de acesso aos serviços públicos.

Em um estudo baseado em uma amostra da população dos Estados Unidos da América (EUA), quase metade dos participantes relatou ter necessidades de saúde bucal não atendidas após o diagnóstico de HIV ${ }^{22}$. No Mississipi, a atenção à saúde bucal e o exame oral foram as necessidades não atendidas com maior frequência entre as $\mathrm{PVHA}^{23}$. Contudo, é preciso compreender que a comparação entre Brasil e EUA não pode ser direta, haja vista uma discrepância entre os modelos de atenção à saúde utilizados nos dois países. Enquanto o Brasil possui um sistema público, universal e gratuito, que engloba a saúde bucal, os EUA possuem um sistema privado, acessado pela maioria da população via seguros de saúde, quem nem sempre incluem saúde bucal no rol de procedimentos disponíveis ${ }^{24}$. Assim, é compreensível o baixo acesso dos pacientes PVHA entre os norte-americanos, o que não seria tão previsível no sistema brasileiro.

Em uma amostra de pacientes nigerianos, as necessidades não atendidas foram relatadas por $79,4 \%{ }^{25}$. Estudo brasileiro demonstrou uma alta demanda por serviços de atenção à saúde bucal em PVHA ${ }^{16}$. Por outro lado, a participação em programas governamentais voltados à melhoria do acesso à atenção à saúde bucal foi associada ao bem-estar geral em PVHA de baixa renda ${ }^{\mathbf{2 6}}$, o que deveria incentivar esses pacientes a buscarem atenção à saúde bucal. No entanto, neste estudo, o uso de serviços públicos gratuitos de saúde foi limitado, pois apenas um quarto da amostra relatou que conseguiu atendimento no CSF/CEO.

Entendendo a importância da atenção à saúde bucal na qualidade de vida das PVHA, buscou-se compreender como ela está sendo realizada no grupo pesquisado. Os resultados revelaram que mais de um terço dos entrevistados não se consultou com o dentista nos últimos dois anos, e que, dos que foram atendidos, mais da metade fez tratamento odontológico utilizando serviços privados. Nesse contexto, é importante mencionar que 
os achados de Cavalcante et al. ${ }^{27}$ e Araújo ${ }^{16}$ ressaltam que $21,0 \%$ e $45,1 \%$ dos usuários, respectivamente, afirmaram ter consultado um cirurgião-dentista há menos de um ano; enquanto em um estudo nos EUA, a maioria dos pacientes $(52,4 \%)$ não havia consultado há mais de dois anos, $48,2 \%$ relataram uma ou mais necessidades orais não satisfeitas após o diagnóstico do HIV e $63,2 \%$ classificaram a saúde de seus dentes e gengivas como 'regular' ou 'ruim'27. Tendo em vista que os custos de serviços odontológicos particulares são altos e que a população estudada, em sua maioria, é de nível socioeconômico baixo, evidencia-se, assim, o interesse das PVHA na consulta odontológica e uma baixa acessibilidade dos serviços públicos odontológicos a essa população. Isso destaca a importância do desenvolvimento de estratégias para reduzir custos, aumentar o uso e reduzir as barreiras pessoais à atenção à saúde bucal, principalmente considerando o impacto da saúde bucal precária nessa população ${ }^{22}$.

Adiciona-se a preocupação da dificuldade de acesso aos serviços de saúde por parte dessa população, o baixo nível de atividades de educação em saúde desenvolvida pela ESB às PVHA. Mais de dois terços dos usuários do SUS que foram atendidos nos CSF/CEO não participaram dessa atividade educativa, resultados concordantes com outros estudos ${ }^{16,27}$. Essa descoberta é preocupante, haja vista que a atenção em saúde bucal impacta a qualidade de vida das PVHA $^{28}$, principalmente quando apoiadas pela educação em saúde para a prevenção de doenças periodontais, bem como infecções bucais oportunistas ${ }^{2}$.

No entanto, ter acesso ao serviço público de atenção à saúde bucal não é sinônimo de resolutividade/conclusão do tratamento. Quase metade dos usuários relatou não ter finalizado o tratamento odontológico. A falta de materiais, os problemas nos equipamentos ou as reformas nos CSF foram os motivos mais citados pelas PVHA que não completaram tratamento odontológico. Essas dificuldades, com a superlotação e a falta de vagas no CSF, foram descritas por quase a totalidade dos usuários que tentaram, mas não conseguiram ser atendidos nos serviços municipais. Esses achados indicam um sério problema de acesso e resolutividade no atendimento às PVHA que buscam atenção à saúde bucal pública em Fortaleza (CE). Araújo ${ }^{16}$ destaca a falta de vagas nos serviços públicos como o principal problema das PVHA para conseguir acesso à consulta odontológica, apontado por $46,2 \%$ dos usuários. Além disso, esse autor ressalta a recusa dos profissionais ao atendimento como um relevante incomodo. Isso pode explicar a alta porcentagem de pacientes que omitem sua condição de HIV positivo para os profissionais de odontologia 23 .

A discriminação, ou o receio desta, pode induzir as PVHA a se autodiscriminarem na busca da assistência odontológica, fazendo com que elas escondam sua soropositividade e/ou procurem tratamento longe de seu domicílio, onde, teoricamente, são menos conhecidas, e, consequentemente, possuem menor vínculo com o serviço e maior dificuldade de acesso. Corroborando os resultados da literatura ${ }^{16,17,23,25-27}$, a presente investigação demonstrou que mais de um terço dos usuários que tiveram acesso ao CSF/CEO foram atendidos longe do bairro em que residem; e, destes, quando encaminhados pelo SAE para o cirurgião-dentista, quase a metade solicitou atendimento longe da sua residência.

Pesquisas evidenciaram que mais da metade das PVHA não revelou ao cirurgião-dentista sua condição sorológica ante o tratamento odontológico. As principais justificativas dessas pessoas para omitir seu estado na condição de HIV positivo podem consistir no medo de recusa do atendimento e no receio de serem discriminadas ou tratadas com preconceito ${ }^{16,17}$. Igualmente, na presente investigação, quase um quarto dos usuários atendidos afirmou já ter omitido alguma vez a condição de HIV positivo durante o atendimento odontológico. Quase $90 \%$ apontaram como motivos o medo de ter atendimento recusado ou serem discriminados. 
As dificuldades para acessar o serviço podem ser consequência da vulnerabilidade e do preconceito que esse grupo sofre. Também podem estar relacionadas com a precária formação humanística e técnica dos profissionais da saúde; com a forma do processo de trabalho das EqSF, que não favorecem a criação de vínculo com o usuário e o território vivo; com a não priorização de grupos vulneráveis, o que pode permitir que a discriminação (explícita ou implícita) se instale nos CSF; com a estrutura social, cultural, econômica e política da sociedade. Essas questões podem ser consideradas como violência estrutural às PVHA, a qual se expressa nas oportunidades desiguais, na discriminação e na injustiça, como, por exemplo, no acesso à educação e a serviços de saúde. Johan Galtung ${ }^{29}$ a define como uma violência que não é praticada por um agente concreto com o objetivo de infligir sofrimento, mas é gerada pela própria estrutura social. A violência estrutural não é, necessariamente, um processo ativo e deliberado, podendo revelar-se pela ausência de proteção e de garantia de direitos e necessidades. Ela está intimamente ligada à injustiça social, já que afeta as pessoas de maneiras diferentes dentro das diversas estruturas sociais ${ }^{30}$. Esses aspectos levam, então, a refletir sobre a importância de aplicação dos princípios da ética e da bioética no atendimento das PVHA, visto que, ambientes discriminatórios e preconceituosos, que geram invasão de privacidade, dificultam a assistência a esse grupo de usuários ${ }^{31}$, e podem comprometer seu acesso e sua adesão ao tratamento, afetando sua saúde.

Considerando todas essas questões em discussão, evidenciou-se, também, que, para efetivação de uma atenção à saúde bucal resolutiva e contínua, é necessária, além de amplo acesso e boa qualidade do serviço, a adesão do usuário ao tratamento. Para tanto, a prática do cuidado humanizado às PVHA, por parte da ESB, pode contribuir de maneira significativa, apoiando esses usuários na continuidade do tratamento. A humanização da atenção deve ser efetivada no cotidiano dos serviços, concretizando uma boa relação profissional-paciente e buscando a melhoria da qualidade de vida dos usuários ${ }^{32}$. Tecnicamente, humanização é sinônimo de assistência de qualidade, associada ao reconhecimento da subjetividade, direitos e referências culturais dos pacientes ${ }^{33}$. Nesse sentido, a humanização da atenção busca alterar as relações entre trabalhadores e usuários e dos trabalhadores entre si, estabelecendo vínculo/ responsabilização das equipes com os usuários. A autonomia deve ser respeitada, considerando sua singularidade, e, em se tratando da atenção às PVHA, sua vulnerabilidade, levando em conta a subjetividade da doença ${ }^{32,33}$.

Sobre as práticas de cuidado humanizado observadas durante a consulta das PVHA, verificou-se, no presente estudo, nota de 7,6 $( \pm 2,5)$ atribuída pelos pacientes ao atendimento prestado pelo cirurgião-dentista. A maioria dos usuários entrevistados acredita que os profissionais que os atenderam estão preparados para resolver seus problemas de saúde bucal, declarando-se muito satisfeitos ou satisfeitos em relação ao grau de satisfação geral do atendimento. Mais de $70 \%$ das PVHA atendidas nos CSF/CEO assinalaram muito satisfeitos ou satisfeitos, considerando o respeito, a atenção e o repasse de informações de forma clara durante o atendimento.

Em contraste com estudo anterior ${ }^{34}$, na presente investigação, quando perguntado às PVHA atendidas nos CSF/CEO se já haviam passado, durante $o$ atendimento odontológico, por alguma forma de discriminação e/ou preconceito, ou ainda se haviam observado algum tipo de resistência por parte dos profissionais em atendê-las, apenas um percentual muito baixo respondeu que sim. Esse achado é relevante, pois comprova que, ao menos sob a ótica das PVHA atendidas, a ESB tem conseguido oferecer assistência humanizada, considerando os resultados discutidos. Ou seja, os profissionais que se dispõem a atender as PVHA o fazem de forma humanizada e desprovida de preconceito.

É interessante enfatizar, como já discutido anteriormente, que a maior adesão do usuário 
ao tratamento passa por várias questões, que vão desde a acessibilidade (por exemplo, distância da unidade de saúde de sua residência, disponibilização de horários para seu atendimento), passando pela presença de material odontológico em quantidade e qualidade necessários, profissionais qualificados para $o$ atendimento, aptos a acolher e a resolver as demandas do paciente, até questões mais subjetivas, como humanização do cuidado e criação de vínculo paciente-profissional. Dessa forma, é necessário que políticas públicas voltadas ao cuidado desses pacientes compreendam e trabalhem esses aspectos, enfocando a formação profissional, a organização da rede de atenção, a qualificação do processo de referência e contrarreferência, a estruturação dos serviços de saúde bucal, entre outros aspectos.

\section{Considerações finais}

Pode-se concluir que o atendimento odontológico humanizado às PVHA nos CSF/CEO de Fortaleza (CE) pode ser uma ferramenta fundamental no processo de adesão do usuário ao tratamento, bem como sua importância na avaliação desses serviços. Assim, a metodologia utilizada no presente estudo permitiu uma descrição da realidade, com grande parte dos usuários declarando-se satisfeita com a qualidade do serviço odontológico prestado pelas ESB, embora tenham sido observadas queixas quanto à interrupção do tratamento ocasionadas pela deficiência de recursos físicos e materiais. Evidencia-se, portanto, que a atenção à saúde bucal às PVHA em Fortaleza (CE) é de difícil acesso e possui entraves nos procedimentos de agendamento e encaminhamento dos pacientes. Os profissionais dispostos a tratar as PVHA foram percebidos como oferecendo um nível satisfatório de atendimento, embora os avanços significativos no acesso a medicamentos antirretrovirais e do estabelecimento de centros de referência para as PVHA, os serviços públicos de saúde integrados - incluindo atenção à saúde bucal - ainda estejam em estágio inicial.

O presente estudo esclareceu a importância do atendimento integrado às PVHA, sendo necessária a distribuição de medicamentos antirretrovirais, que se mostra insuficiente para apoiar totalmente a qualidade de vida desses pacientes. Outrossim, esta pesquisa pode contribuir para uma relevante discussão a respeito dessas questões enquanto levadas em consideração pelos sistemas que pretendem enfrentar o desafio de cuidar das PVHA.

\section{Colaboradores}

Maia LA (0000-0003-0634-1757)*, VieiraMeyer APGF (0000-0003-4237-8995)*, Saintrain MVL (0000-0003-1514-3218)*, Nuto SAS (0000-0002-4763-6773)* e Morais APP (0000-0001-6188-7897)* colaboraram igualmente para concepção e planejamento da pesquisa; para análise e interpretação dos dados e para redação e aprovação da versão final do manuscrito. 


\section{Referências}

1. World Health Organization. Global health sector strategy on HIV/AIDS 2011-2015. Geneva: World Health Organization; 2011.

2. Buchala CM, Cavalheiro TR. A classificação internacional de funcionalidade, incapacidade e saúde e a aids: uma proposta de core set. Acta Fisiatrica. 2008; 15(1):42-8.

3. Brasil. Ministério da Saúde. Boletim epidemiológico HIV/ AIDS. Brasília, DF: Ministério da Saúde; 2019 [acesso em 2020 jan 10]. Disponível em: http://www.aids.gov. br/pt-br/pub/2019/boletim-epidemiologico-de-hivaids-2019.

4. Report on the global AIDS epidemic. Relatório Informativo. Geneva: UNAIDS 2019. [acesso em $2019 \mathrm{dez} 6$ ]. Disponível em: https://unaids.org.br/wp-content/uploads/2019/11/2019_UNAIDS_WAD2019_FactSheet.pdf.

5. Naidoo P. Barriers to HIV Care and Treatment by Doctors: A review of the literature. S Afr fam pract. 2006; 48(2):55-16e.

6. Brasil. Lei $\mathrm{n}^{\circ}$ 9.133, de 13 de novembro de 1996. Dispõe sobre a distribuição gratuita de medicamentos aos portadores do HIV e doentes de AIDS. Diário Oficial da União. 14 Nov 1996. [acesso em 2019 set 16]. Disponível em: http://www.planalto.gov.br/ccivil_03/leis/19313. htm.

7. Silva KL, Sena RR, Grillo MJC, et al. Formação do enfermeiro: desafios para a promoção da saúde. Esc Anna Nery Rev Enferm. 2010 [acesso em 2012 set 12]; 14(2):368-376. Disponível em: http://www.scielo.br/pdf/ean/v14n2/21. pdf.

8. Mooneya B, Timminsb F, Byrneb G, et al. Nursing students' attitudes to health promotion to: Implications for teaching practice. Nurse educ. today. 2011 [acesso em 2013 ago 15]; 31(8):841-848. Disponível em: http:// www-sciencedirect-com.ezl8.periodicos.capes.gov.br/ science/article/pii/S026069171000256X\#bb0255.

9. Czeresnia D. O Conceito de Saúde e a diferença entre Prevenção e Promoção. In: Czeresnia D, Freitas CMF, organizadores. Promoção da Saúde, conceitos, reflexões, tendências. Rio de Janeiro: Fiocruz; 2003.

10. Coogan MM, Greenspan J, Challacombe SJ. Oral lesions in infection with human immunodeficiency virus. Bull World Health Organ. 2005; 83(9):700-706.

11. Barros AJD, Santos IS, Bertold ID. Can mothers rely on the Brazilian health system for their deliveries? An assessment of the use of public system and out of- pocket expenditure in 2004 Pelotas Birth Cohort Study, Brazil. BMC Health e Serv Res. 2008; (8):57-63.

12. Borges MJL. Integralidade da atenção à saúde das pessoas vivendo com HIV/Aids: uma avaliação de serviços de assistência. [dissertação]. Recife: Fundação Oswaldo Cruz; 2010. 137 p.

13. Giovanella L. Atenção Primária à Saúde: seletiva ou coordenadora dos cuidados? Rio de Janeiro: Cebes; 2008.

14. Brasil. Ministério da Saúde. Diretrizes da política nacional de saúde bucal [internet]. Brasília, DF: Ministério da Saúde; 2004. [acesso em 2019 dez 6]. Disponível em: http://dab.saude.gov.br/portaldab/biblioteca. php?conteudo=publicacoes/pnsb.

15. Brasil. Ministério da Saúde. Portal Brasil. Informação e Gestão da Atenção Básica. Histórico de Cobertura. 2019. [acesso em 2019 dez 5]. Disponível em: https://egestorab.saude.gov.br/paginas/acessoPublico/relatorios/relHistoricoCoberturaConsolidado.xhtml.

16. Araújo DB. Condições de saúde bucal e utilização de serviços odontológicos por pessoas que vivem com HIV/ AIDS no município de Fortaleza-Ceará. [dissertação]. Fortaleza: Universidade Federal do Ceará; 2010. 110 p. [acesso em 2019 set 7]. Disponível em: http://www.repositorio.ufc.br/bitstream/riufc/1011/1/2010_dis_dbaraujo.pdf.

17. Pires MBO, Martelli Júnior H, Melo Filho MR, et al. Dificuldades percebidas por pacientes vivendo com HIV/ AIDS em obter tratamento odontológico. Arq Odontol. 2008; 44(3):25-31. 
18. Ceará. Secretaria de Saúde do Estado. Boletim Epidemiológico HIV/Aids. Fortaleza; 2019. [acesso em 2020 jan 12]. Disponível em: saude.ce.gov.br/wp-content/uploads/ sites/9/2018/06/BOLETIM_-AIDS_-2019_29_11 2019. pdf.

19. Donabedian A. The quality of care. How can it be assessed? JAMA. 1988; 260(12):1743-1748.

20. Jones J, Mofidi M, Bednarsh $\mathrm{H}$, et al. Increasing access to oral health care for people living with HIV/AIDS in rural Oregon. Public Health Rep. 2012; 127(supl2):65-72.

21. Fox JE, Tobias CR, Bachman SS, et al. Increasing access to oral health care for people living with HIV/AIDS in the U.S.: baseline evaluation results of the Innovations in Oral Health Care Initiative. Public Health Rep. 2012; 127(supl2):5-16.

22. Jeanty Y, Cardenas G, Fox JE, et al. Correlates of unmet dental care need among HIV-positive people since being diagnosed with HIV. Public Health Rep. 2012; 127(supl2):17-24.

23. Krause DD, May WL, Butler Jr KR. Determining unmet, adequately met, and overly met needs for health care and services for persons living with HIV/AIDS in Mississippi. AIDS Care. 2012; 25(8):973-979.

24. Noronha JC, Ugá MAD. O sistema de saúde dos Estados Unidos. In: Buss PM, Labra ME, organizadores. Sistemas de saúde: continuidades e mudanças. Rio de Janeiro: Editora Fiocruz; 1995. 265 p. [acesso em 2019 set 30]. Disponível em: http://books.scielo.org.

25. Adedigba MA, Ogunbodede E, Jeboda SO, et al. Self-perceived and unmet general health need among PLWHA in Nigeria. East Afr J Public Health. 2008; 5(3):199-204.

26. Bachman SS, Walter AW, Umez-Eronini A. Access to oral health care and self-reported health status among low-income adults living with HIV/AIDS. Public Health Rep. 2012; 127(supl2):55-64.

27. Cavalcante CAT, Gonçalves DHA, Maia DKA, et al. A atenção em saúde bucal para DST/HIV/AIDS na rede municipal de Belo Horizonte: diagnóstico loco-regional. Belo Horizonte: Secretaria Municipal de Saúde; 2006. [acesso em 2015 ago 18]. Disponível em: http://www. pbh.gov.br/smsa/biblioteca/saudebucal/atencaoemsaudebucalparadsthivaids.pdf.

28. Brown JB, Rosenstein D, Mullooly J, et al. Impact of intensified dental care on outcomes in human immunodeficiency virus infection. AIDS Patient Care STDS. 2002; 16(10):479-86.

29. Galtung J. Violence, Peace, and Peace Research. J Peace Res. 1969; 6(3):167-191.

30. Farmer PE, Nizeye B, Stulac S, et al. Structural Violence and Clinical Medicine. PLOS Med. 2006; 3(10):16861691.

31. Carneiro HG. Ética e Bioética no atendimento aos pacientes portadores de HIV/aids no Sistema Único de Saúde. Cad IberAmer Direito Sanit. 2013; 2(2):839-849.

32. Campos GWS. Humanização na saúde: um projeto em defesa da vida? Interface (Botucatu). 2005 [acesso em 2019 ago 27]; 9(17):398-403. Disponível em: http://www. scielo.br/pdf/icse/v9n17/v9n17al6.pdf.

33. Deslandes SF. Análise do discurso oficial sobre a humanização da Assistência hospitalar. Ciênc. Saúde Colet. 2004; 9(1):7-14.

34. Andrade JT, Holanda VMS, Gondim APS. Em busca do humano: avaliação do Humaniza SUS em ações municipais de saúde em Fortaleza. Relatório Final. Observatório de Recursos Humanos em Saúde - CETREDE / UFC; Fortaleza: Núcleo de Pesquisa em Educação e Saúde Coletiva - UECE; 2007. [acesso em 2015 set 30]. Disponível em: http://www.observarh.org.br/observarh/ repertorio/Repertorio_ObservaRH/CETREDE/Busca humano.pdf.

Recebido em 15/03/2020

Aprovado em 05/11/2020

Conflito de interesses: inexistente

Suporte financeiro: não houve 